\title{
Study on Co-Simulation Technology Based on ADAMS and MATLAB
}

\author{
Hu Zhongling ${ }^{1, *}$, Xu Dongsheng ${ }^{2}$, Liu Guangsheng ${ }^{1}$, Jia Changzhi ${ }^{1}$ \\ ${ }^{1}$ Department of Guns Engineering, Ordnance Engineering College, 050003 Shijiazhuang, China \\ ${ }^{2}$ College of Field Engineering, PLA Univ. of Sci. \&Tech, 210007 Nanjing, China
}

\begin{abstract}
Co-simulation technology has been widely used in the design and research of complex mechanical systems. The co-simulation based on ADAMS and MATLAB is summarized, and the related software is introduced. Taking eccentric connecting rod as an example, the output torque of MATLAB is the input parameter of mechanical model, and the control effect under three kinds of outputs is compared. The results show that the co-simulation based on ADAMS and MATLAB can be used as an effective method for the simulation analysis of complex dynamic systems.
\end{abstract}

\section{Introduction}

In the field of computer numerical simulation, with the further development of computer simulation technology and its application, the scale and complexity of the research object is increasing rapidly. The application of single machine, control, hydraulic or other software has been difficult to meet the needs of the development of the research object. Co-simulation analysis has become one of the key technologies to improve the performance of the product.

In the co-simulation analysis, the connection between different software is established, so the model built in a software can take its calculation results as system input signals to the model built in another software. This instruction includes the force, torque, driving and other typical signals. The latter model can generate the relevant response, such as displacement, velocity, acceleration and so on. So the simulation data can be transmitted between different simulation software.

The combined simulation analysis has been applied in many fields, the multi-body dynamics and control system simulation is one of the most typical application. ADAMS and MATLAB are the most popular software in the simulation of multi-body dynamics and control system. This paper summarizes the application and development of ADAMS and MATLAB, put forward the key problems in the co-simulation process, finally do further clarification through the example of Eccentric connecting rod.

\section{ADAMS and MATLAB}

\subsection{ADAMS}

ADAMS software is a mechanical system dynamics simulation analysis software developed by American MDI company, it uses graphical environment and parts library, binding library, force database, create a fully parametric geometric model of the mechanical system, the solver using the Lagrange equation from multi rigid body system dynamics theory, for which the dynamic equations of the system are established, the statics, kinematics and dynamics analysis for the virtual machine system are achieved, finally output displacement, velocity, acceleration and reaction curve. ADAMS software simulation can be used to predict mechanical system performance, range of motion, collision detection, peak load and can be the input load to calculat finite element etc.

ADAMS/Controls is an integrated module in the ADAMS software package. In ADAMS/Controls, designers can design a simple control mechanism, or use general control system software (such as MATLAB, EASYS) to establish a control system block diagram, build a simulation model including the control system, hydraulic system, pneumatic system and mechanical motion system.

\subsection{MATLAB}

MATLAB is a high-level technical computing language and interactive environment for the development of algorithms, data visualization, data analysis and numerical calculation. By the use of MATLAB, the engineering calculating problems can be solved faster than by the traditional programming languages (such as C, $\mathrm{C}++$ and FORTRAN).

The application of MATLAB is very wide, including signal and image processing, communication, control system design, testing and measurement, financial modeling and analysis, computational biology, and many other applications. The additional toolbox (provided by a dedicated MATLAB function set) extends the MATLAB environment in order to solve the specific type of problem in these application domains.

Simulink is a very powerful dynamic system modeling and simulation software package for MATLAB, which provides a complete and flexible visual design and debugging environment for the user's modeling and simulation process, and contains a wealth of basic functional modules and many professional fields.

Simulink provides a graphical user interface (GUI) to build dynamic system model. This process can be accomplished by clicking and dragging the mouse operation. Especially for complex system modeling, it can be faster, and users can immediately see the system simulation results. Simulink also provides a virtual reality toolbox, user can use a mathematical model to directly drive the geometric model, and to make the simulation more vivid and realistic. 


\section{Co-simulation based on ADAMS and MATLAB}

\subsection{Overview of co-simulation applications}

The modern mechanical system almost contains one or more control systems, most of which may include hydraulic, electronic, pneumatic, hydraulic and other systems, the control system contained in these systems may greatly affect the performance. In the traditional research and design process of mechanical and electrical integration, mechanical engineer and control engineer both work on the design and development of a system, but they all need to build their own model. And then use different analysis software, to do independent design, debugging and experiment. Finally, do joint commissioning. If they found some problems, they go back to their respective model, and then combined. Obviously this way is time-consuming and laborious.

Through modeling and co-simulation technology based on multi domain, this problem is well solved. This technology provides a new design method for mechanical and control system. Mechanical engineer and control engineer can enjoy the same prototype model to design, debug and test. They can use the virtual prototype of the mechanical system and control system for repeated joint commissioning, until they get a satisfactory design results, and then do the construction and commissioning of the physical prototype.

For the design engineer of control system, ADAMS/Controls can integrate the motion of the mechanical system and the simulation of the control system. The mechanical system not only includes the quality of the components, but also can consider the friction, gravity, and other factors. ADAMS solver can be used to solve the model, or by using the control software. The calculation results will be the complete and precise state of the mechanical system in actual working condition, and the function of the control system is fully reflected.

The co-simulation analysis of mechanical and sontrol system can be used in many fields, for example, automotive anti lock braking system ABS, active suspension, aircraft landing gear booster, satellite attitude control, simulation calculation can be linear, or nonlinear.

ADAMS and ADAMS are widely used in the field of mechanical system simulation and control system simulation. MATLAB provides a powerful modeling and simulation environment for users. Users can model, simulate and analyze various mechanical systems. MATLAB has powerful computing function, high programming efficiency and modular modeling method. Compared with the traditional method, the combined simulation technology has obvious advantages, it can improve the design efficiency, shorten the development cycle, reduce development costs, and optimize the overall performance of the mechanical and electrical integration system.

\subsection{The procedure of co-simulation design}

\subsubsection{Build the ADAMS virtual prototyping model of mechanical system}

ADAMS software can be directly used to model the simple set model. And for the more complex model, it can be carried out in a variety of two-dimensional modeling software, such as Soliworks, UG, etc., then in a certain format into ADAMS. And then the user impose constraints, drive and force, etc.. Before the simulation of mechanical and control system, the simulation analysis of mechanical system is carried out by ADAMS firstly, and then the control system is added after the model of mechanical system is confirmed to be correct.

\subsubsection{Determine the input and output variables of ADAMS}

The input and output data from ADAMS is the communication interface with the MATLAB control system. The output variable in ADAMS is the input variables into the control system; the output variable of the control system is to return to the input variables of ADAMS, and then complete a closed-loop control from ADAMS to MATLAB. The input / output process is shown in Figure 1.

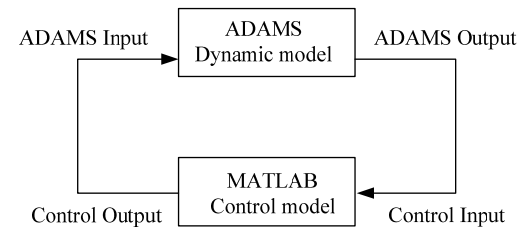

Figure 1. The input/output process between ADAMS and MATLAB

\subsubsection{Control system model}

User can establish a control system model by using MATLAB/Simulink provided by the module library, or writing the $S$ function. Then set the parameters of the various modules and the measurement of the impact. At the same time, the model of the virtual prototype system built in the ADAMS environment is imported into Simulink.

\subsubsection{Combined simulation analysis}

Set the simulation parameters in the Simulink environment, where the simulation step must be consistent with the step size in the ADAMS environment. Then the virtual prototype model is simulated, and the simulation results are given. The mechanical system or the control system is modified according to the need of the whole virtual prototype. 


\section{An example of co-simulation applied to eccentric connecting rod}

\subsection{Eccentric connecting rod model and its input/output}

An eccentric connecting rod was established, and a rotating pair and a single component torque were defined on the connecting rod. The connecting rod would deviate from the horizontal position under the action of gravity. The control object was the torque on the connecting rod. Under the torque's function, the connecting rod would be restored to the starting position. The virtual prototype model is shown in Figure 2.

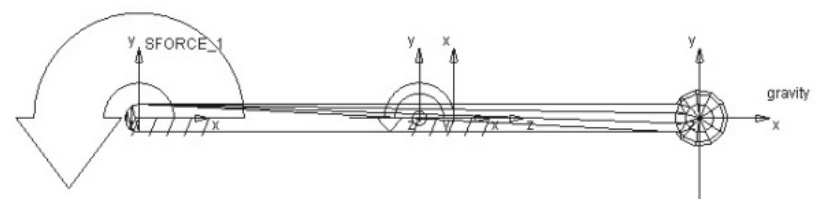

Figure 2. The virtual prototype model of eccentric connecting rod

Plugin Manager command from the menu Tools was selected, which provided several modules for the ADAMS/Aview to load. Controls module was selected. At this point, the Controls/Plant menu in Controls could be seen, and the input and output variables of control system was set up in the ADAMS/Aview Export. The virtual prototype model of eccentric horizontal connecting rod was defined by two output variables: the angle and the angular velocity of the connecting rod, and an input variable: the control torque.

\subsection{Establishment of control model}

In this paper, the most common PID controller was used to control the eccentric connecting rod. The control principle diagram is shown in Figure 3.

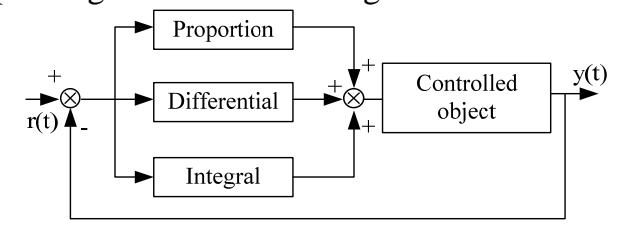

Figure 3. The control principle diagram of PID

PID controller is a linear controller, which is based on $r(t)$ and $e(t)=r(t)-y(t)$, PID control law is:

$$
u(i)=k_{p}\left[e(t)+\frac{1}{k_{i}} \int_{0}^{t} e(\tau) d \tau+\frac{k_{d} d e(t)}{d t}\right]
$$

Where $k_{p}$ is the proportional coefficient, $k_{i}$ is the integration time constant and $k_{d}$ is the differentiating time constant.

Based on the principle of PID controller, this paper established three kinds of control models under different inputs. The three kinds of control models are shown in Figure 4.

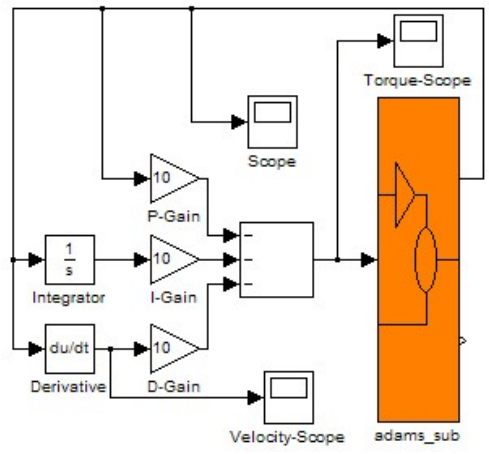

(a) The control model under angle input

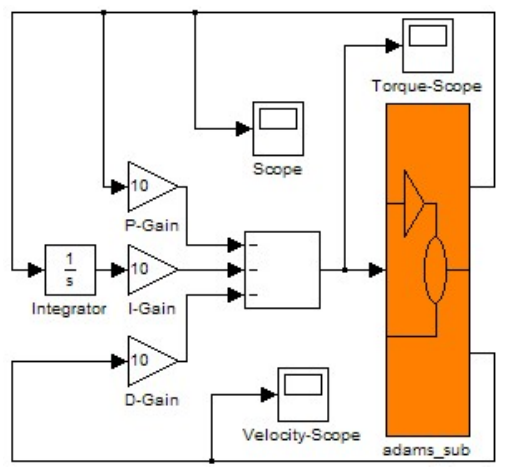

(b) The control model under angle and angular velocity inputs

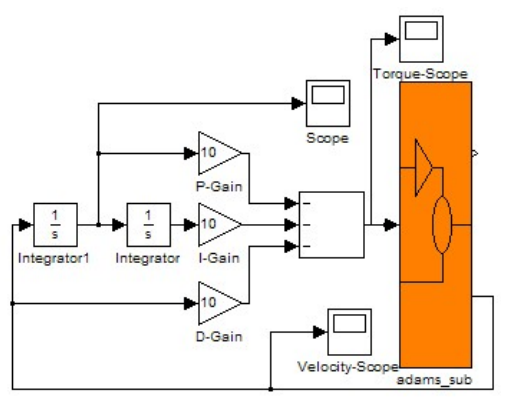

(c) The control model under angle-velocity input

Figure 4. Three kinds of control models under different inputs

\subsection{Co-simulation and results analysis}

After setting up the simulation parameters in the Simulink environment, ran the co-simulation. After each co-simulation, the variation curves of three variables in three cases was compared. Three controllers got same control effects, that was in accordance with the expected results and mathematical reasoning. The variation curves of three state variables are shown in Figure 5.

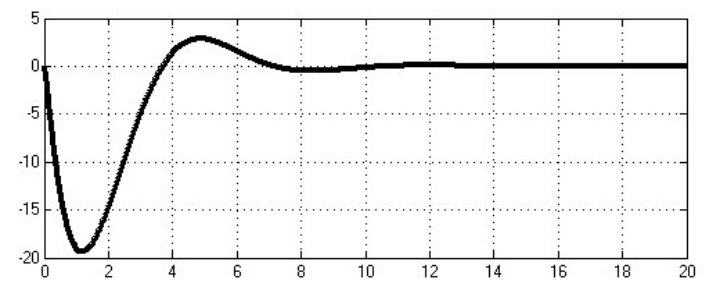

(a) The variation curve of the angle 


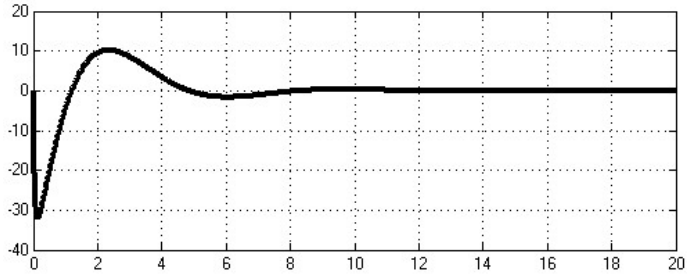

(b) The variation curve of the angular velocity

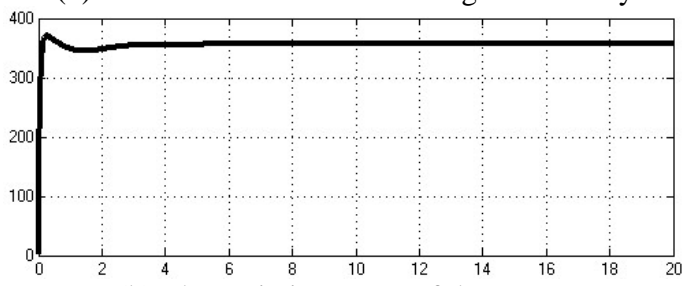

(b) The variation curve of the torque

Figure 5. The variation curves of three state variables

The simulation results showed that the controller designed based on PID can better control the eccentric connecting rod. The connecting rod could quickly reach level and maintain stability. In this paper, the selection of $k_{i}, k_{d}$ and $k_{p}$ in PID controller was directly determined, the optimal parameters could be selected by comparing different parameters, also, applying fuzzy theory and other advanced theories would be effective.

\section{Conclusions}

In this paper, the co-simulation based on the combination of MATLAB and MATLAB was studied, the ADAMS and ADAMS software were introduced, and the characteristics of the combined simulation were summarized.

In the co-simulation, the complex differential equations of mechanical system are not needed, and the virtual prototype model of ADAMS is built up, which greatly facilitates the modeling process. Compared to the simple mathematical model, the virtual model established by ADAMS can be better close to the actual physical model, thus providing a more reliable basis for the follow-up study.

\section{Acknowledgements}

This paper is sponsored by the National Natural Science Foundation of China (Item number: 51175508).

\section{References}

[1] Xiong Guangleng, Guo Bin, Chen Xiaobo. Collaborative Simulation and Virtual Prototyping Technology (Tsinghua University Press, Beijing, 2004)

[2] Li Jianfeng, Wang Jianbing, Lin Jianjun, et al. Case Analysis of Co-Simulation and Integrated Optimization of Mechanical and Electrical Systems (Electronics Industry Press, Beijing, 2010)

[3] Yi Xiushuai, Huang Zhigang, Sun Mingtao. Application of ADAMS and MATLAB Co-
Simulation Technology. J. Journal of Beijing Technology and Business University (Natural Science Edition), 27, 14 (2009)

[4] Wang Tao, Zhang Huiming. Study on Associated Control System Simulation Based on ADAMS and MATLAB Software. J. Mechanical Engineering \& Automation, 6, 80 (2005)

[5] Sun Xiujun, Wang Xiaoyue, Yang Yan. Compendious Introduction of Co-simulation and ADAMS/Controls. J. Mechanical Engineering \& Automation, 1, 62 (2007)

[6] Guan Xiqiang, Qu Qiuzhen, Zhang Jianwu. FourWheel-Steering System Based on The Variable Structure Model Following Control, J. Chinese Journal of Mechanical Engineering, 38, 55 (2001) 